\title{
Teaching NeuroImages: Autoimmune glial fibrillary acidic protein meningoencephalomyelitis
}

Paula K.J. Lee, MD, Juan E. Small, MD, and Michal Vytopil, MD

Neurology ${ }^{\circledR}$ 2019;93:e2076-e2077. doi:10.1212/WNL.0000000000008562

Figure 1 Axial T1 postcontrast brain MRI

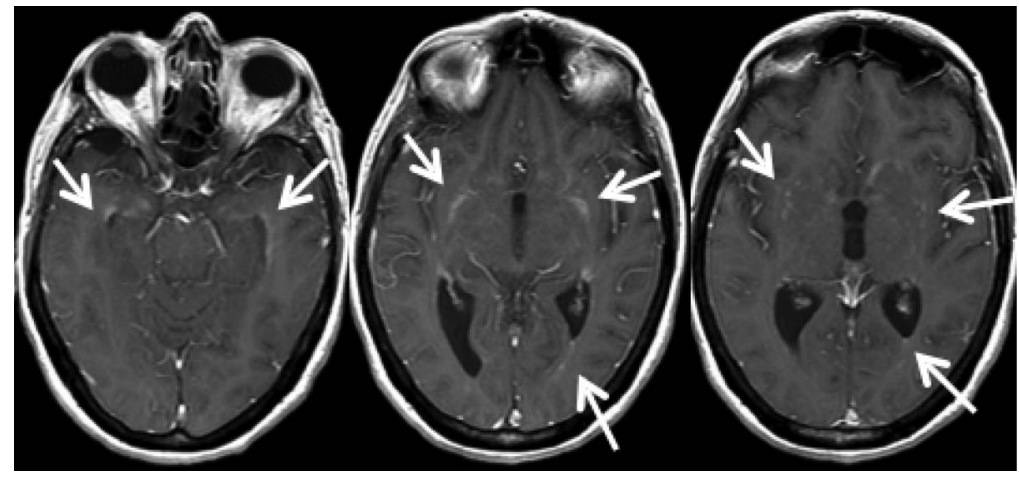

Leptomeningeal

linear/stippled parenchymal, and ependymal postgadolinium enhancement (arrows).

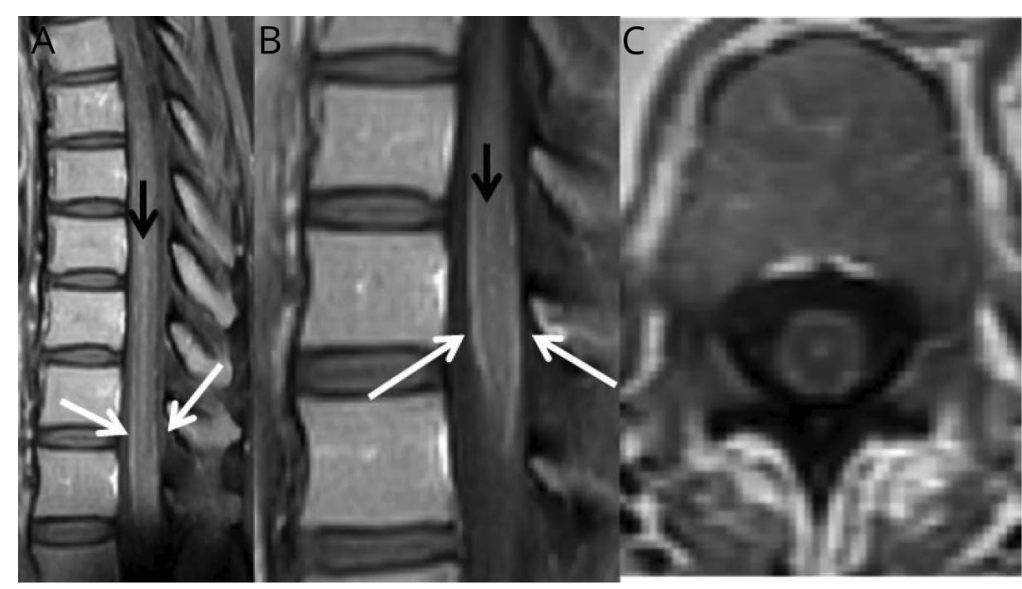

Correspondence

Dr. Lee

clarity1789@gmail.com
Leptomeningeal (white arrows) and central canal enhancement (black arrows) throughout the cord (A) and conus medullaris (B). Axial T1 postcontrast image (C) with peripheral leptomeningeal enhancement and central dot of central spinal canal enhancement.
A 73-year-old woman with myelodysplastic syndrome presented with 2 months of decline in cognition and mobility, accompanied by headache and weight loss. Examination revealed a somnolent woman with symmetric cogwheel rigidity, bradykinesia, arm myoclonus, and hyperreflexia. Brain MRI showed leptomeningeal, linear/stippled parenchymal, and ependymal enhancement (figure 1). Spine MRI demonstrated nonenhancing cervicothoracic cord signal change and circumferential enhancement of conus medullaris with central canal enhancement

\section{MORE ONLINE}

\section{$\rightarrow$ Teaching slides}

links.lww.com/WNL/B2 
(figure 2). CSF was inflammatory with glial fibrillary acidic protein (GFAP)-immunoglobulin G (IgG). The patient responded promptly to IV methylprednisolone. GFAP-IgG identifies a severe but highly corticosteroid-responsive autoimmune meningoencephalomyelitis with distinctive MRI findings. ${ }^{1,2}$

\section{Study funding}

No targeted funding reported.

\section{Disclosure}

The authors report no disclosures relevant to the manuscript. Go to Neurology.org/N for full disclosures.

Appendix Authors

\begin{tabular}{|c|c|c|c|}
\hline Name & Location & Role & Contribution \\
\hline $\begin{array}{l}\text { Paula } \\
\text { K.J. Lee, } \\
\text { MD }\end{array}$ & $\begin{array}{l}\text { Tufts Medical } \\
\text { Center, Boston, MA }\end{array}$ & Author & $\begin{array}{l}\text { Drafted the manuscript for } \\
\text { intellectual content, primary } \\
\text { clinical care of the patient }\end{array}$ \\
\hline
\end{tabular}

Appendix (continued)

\begin{tabular}{llll}
\hline Name & Location & Role & Contribution \\
\hline $\begin{array}{l}\text { Juan E. } \\
\text { Small, } \\
\text { MD }\end{array}$ & $\begin{array}{l}\text { Lahey Hospital } \\
\text { \& Medical } \\
\text { Center, Burlington, } \\
\text { MA }\end{array}$ & Author & $\begin{array}{l}\text { Major role in acquisition of } \\
\text { imaging data, interpreted } \\
\text { the data }\end{array}$ \\
$\begin{array}{l}\text { Michal } \\
\text { Vytopil, } \\
\text { MD }\end{array}$ & $\begin{array}{l}\text { Lahey Hospital \& } \\
\text { Medical Center, }\end{array}$ & Author & $\begin{array}{l}\text { Revised and supervised the } \\
\text { manuscript for intellectual } \\
\text { content, clinical care of the }\end{array}$ \\
& & & $\begin{array}{l}\text { contient } \\
\text { patient }\end{array}$
\end{tabular}

\section{References}

1. Fang B, McKeon A, Hinson SR, et al. Autoimmune glial fibrillary acidic protein astrocytopathy: a novel meningoencephalomyelitis. JAMA Neurol 2016;73: 1297-1307.

2. Flanagan EP, Hinson SR, Lennon VA, et al. Glial fibrillary acidic protein immunoglobulin $\mathrm{G}$ as biomarker of autoimmune astrocytopathy: analysis of 102 patients. Ann Neurol 2017;81:298-309. 


\section{Neurology}

\section{Teaching NeuroImages: Autoimmune glial fibrillary acidic protein meningoencephalomyelitis \\ Paula K.J. Lee, Juan E. Small and Michal Vytopil \\ Neurology 2019;93;e2076-e2077 \\ DOI 10.1212/WNL.0000000000008562}

This information is current as of November 25, 2019

\section{Updated Information \&} Services

References

Subspecialty Collections

Permissions \& Licensing

Reprints including high resolution figures, can be found at: http://n.neurology.org/content/93/22/e2076.full

This article cites 2 articles, 0 of which you can access for free at: http://n.neurology.org/content/93/22/e2076.full\#ref-list-1

This article, along with others on similar topics, appears in the following collection(s):

Autoimmune diseases

http://n.neurology.org/cgi/collection/autoimmune_diseases MRI

http://n.neurology.org/cgi/collection/mri

Information about reproducing this article in parts (figures,tables) or in its entirety can be found online at:

http://www.neurology.org/about/about_the_journal\#permissions

Information about ordering reprints can be found online:

http://n.neurology.org/subscribers/advertise

Neurology ${ }^{\circledR}$ is the official journal of the American Academy of Neurology. Published continuously since 1951, it is now a weekly with 48 issues per year. Copyright @ 2019 American Academy of Neurology. All rights reserved. Print ISSN: 0028-3878. Online ISSN: 1526-632X.

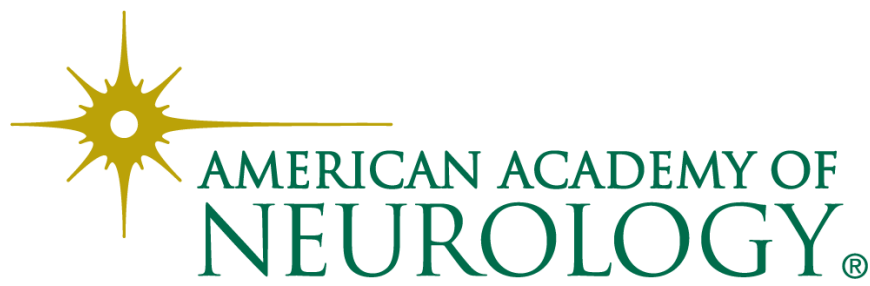

